\title{
XLVI. Molecular frequency and molecular number. Part II. The frequency of the longer residual rays
}

\section{H. Stanley Allen M.A. D.Sc.}

To cite this article: H. Stanley Allen M.A. D.Sc. (1918) XLVI. Molecular frequency and molecular number. Part II. The frequency of the longer residual rays, Philosophical Magazine Series 6, 35:209, 404-409, DOI: $10.1080 / 14786440508635779$

To link to this article: http://dx.doi.org/10.1080/14786440508635779

曲 Published online: 08 Apr 2009.

Submit your article to this journal $\lceil\pi$

Џ Article views: 3

Q View related articles 
and passes through the point $-\imath \sec \alpha$. Now combining (22) and (24), the resultant of all the pressures is seen to reduce to a couple of unoment $-\frac{1}{2} \pi \rho \sin ^{2} \alpha \sin 2 \beta$.

Introducing the velocity and radius, we have

$$
\text { Couple }=-\frac{1}{2} \pi \rho \alpha^{2} \mathrm{U}^{2} \sin ^{2} x \sin 2 \beta . \quad \text {. . }
$$

This hecomes zero in the case of the complete cylinder, $\alpha=\pi$, and reduces, in the case of a plane, to

$$
-\frac{1}{2} \pi \rho b^{2} \mathrm{U}^{2} \sin 2 \beta, \text {. . . . . }
$$

which are known to be correct results.

$\S 8$. In (23) above, it follows that if $\beta=\frac{\alpha}{2}$, there is no term in $\zeta^{\frac{1}{2}}$. This corresponds to the case in which the stream-line $\psi=0$ divides at the elge of the lamina. If this may be applied to the case of an aeroplane wing, it is seen to lead to the conclusion that the eatering edge should point downwards, at an angle equal to the angle of attack of the chord of the (cambered) plane. Observation and photographs would seem to show that in practice snch a condition has been found most favourable.

P.S.-Since the majority of the above results were obtained, a paper has appeared (Dr. J. G. Leathem, Phil. Mag. (6) xxxv. Jan. 1918) in which it is shown by a general method, that (14) above is deducible from (6), and, in fact, when applied, does give the result (14) obtained independontly above.

Loughborough, Fub. 5th, 1918.

XLVI. Molecular Frequency and Molecular Number. Part II. The Frequency of the Longer Residual Rays. By H. Stanley Allen, M.A., D.Se., Eniversity of London, King's College*.
A METHOD of studying the frequency of vibration of A the atoms in compounds is afforded by the "residual rays" obtained by repeated reflexions from the surfaces of solids, and studied by Rubens and his collaborators. It may be assumed that the frequency of such infin-red radiation corresponds with the frequency of vibration of an electrically clarged ion, and, in certain cases at least, it is to be expected that the ion in question may be identified with one of the
* Comminicated by the Author. 
atoms contained in the compound. The writer has shown* that the characteristic frequency, $\nu$, of the atom of a solid element is connected with the atonic number $\mathrm{N}$ by the relation

$$
\mathrm{N} \nu=n \nu_{\Lambda} \quad \text { or } \quad \mathrm{N} \nu=\left(n+\frac{1}{2}\right) \nu_{\Lambda},
$$

where $n$ is an integer, and $\nu_{\mathrm{A}}$ a constant frequency having a value not far from $21 \times 10^{12}$ sec. $^{-1}$. Seeing that the forces which control the vibrations of the atoms in solids are the same whether it be a question of the specific heat of the solid or the reflexion of radiation, it might be anticipated that the same relation would hold in connexion with the residual rays.

According to the theory developed by Nernst + the heat energy of a compound in the solid state is made up of the energy due to the motion of the molecules relative to one another and that due to the vibrations of the atoms in the molecule. The first contribution is calculated by the fornula of Debye, the second by the formula of Einstein. Each calculation involves a knowledge of the corresponding characteristic frequency; the first, $\nu_{1}$, is given, approximately at least, by Lindemann's formula; the second, $v_{2}$, is found from the residual rays of Rubens. Nernst has pointed out that in the case of $\mathrm{KCl}$ and $\mathrm{NaCl}$, the value of $\nu_{1}$ is almost 0.75 times the valne of $\nu_{2}$; and in other cases, although the molecular frequency is slower than the internal frequency, the two are not very different. Indeed, in his earlier work Nernst obtained fairly good agreement by calculating the values of the atomic heat on the assumption of only one frequency, $\nu$. The writer has shown $\ddagger$ that the molecular frequency is connected with the "molecular number" by a relation of the same form as that already quoted for the atomic frequency and the atomic number. The collective results suggest that the internal frequency may conform to the same relation. In the following pages the experimental results are examined for a number of compounds, and it is found that there is a strong presumption in favour of the establishment of the relation suggested.

$\mathrm{NaCl}$. In the case of rock salt strengths were observed, a stronger at $53.6 \mu$ and a weaker at $46.9 \mu$. The corresponding frequencies are $5.60 \times 10^{12}$ and $6.10 \times 10^{12}$ sec. $^{-1}$. Assuming the former

* H. S. Allen, Roy. Soc. Proc. vol. xciv. p. 100 (1917) ; Phil. Mag. vol. xxxiv, p. 478 (1917).

$\dagger$ Nernst, 'The 'Theory of the Solid State' (London, 1914).

$\ddagger$ H. S. Allen, Phil. Mag. vol, xxxv., April 1918.

$\$$ Rubens and Hollnagel, Preuss. Aliad. Berlin, vol. iv. p. 26 (1910).

Phil. Mag. S. 6. Vol. 35. No. 209. May 1918. 
to correspond with the atom of sodium $(N=11)$, and the latter with the atom of chlorine $(\mathrm{N}=17)$, we find for the sodium atom $\mathrm{N}_{\nu}=3 \times 20.5 \times 10^{12}$ and for the chlorine atom $\mathrm{N} \nu=5 \times 21.7 \times 10^{12}$. If, however, we associate the ehlorine atom with the stronger line and the sodium atom with the weaker, we obtain for the products $95.1 \times 10^{12}$ and $70.4 \times 10^{12}$, which are not integral multiples of $21 \times 10^{12}$.

zcl. For sylvin $\nmid$ similar results are found, but in this case the stronger line $\left(62 \cdot 0 \mu\right.$ or $4 \cdot 84 \times 10^{12}$ sec. $\left.^{-1}\right)$ (must be associated with chlorine, the weaker $\left(70.3 \mu\right.$ or $4.27 \times 10^{12}$ sec. $\left.^{-1}\right)$ with potassium $(\mathrm{N}=19)$. In this case we find $\mathrm{N} \nu=4 \times 20.6 \times 10^{12}$ and $\mathrm{N} \nu=4 \times 20.3 \times 10^{12}$ respectively. The products obtained when the atoms are interchanged are $91.9 \times 10^{12}$ and $72 \cdot 6 \times 10^{12}$, which are not integral multiples of $21 \times 10^{12}$.

$\mathrm{KBr}$. The stronger line* is at $86.5 \mu\left(\nu=3.47 \times 10^{12} \mathrm{sec}^{-1}\right)$, the weaker at $75.6 \mu\left(\nu=3.97 \times 10^{12}\right.$ sec. $\left.^{-1}\right)$. The former must be associated with the potassium atom, giving $\mathrm{N} \nu=3 \times 21.9 \times 10^{12}$, the latter with the bromine atom, giving $\mathrm{N} \nu=6 \frac{1}{2} \times 21.4 \times 10^{12}$. The occurrence of $\frac{1}{2}$ in the latter case is noteworthy, as it is supported by independent evidence in connexion with specific heats.

It may be noted that for these three compounds the stronger line is associated with the element of smaller atomic number.

KI. The residual radiation has not been separated into two rays of dlfferent strengths, but it is stated by the experimenters + that such a constitution is possible. In such a case we miay call $\nu_{1}$ and $\nu_{2}$ the frequencies corresponding to elements having atomic numbers $\mathrm{N}_{1}$ and $\mathrm{N}_{2}$, and put

By addition,

$$
\mathrm{N}_{1} \nu_{1}=n_{1} \nu_{\Lambda}, \quad \mathrm{N}_{2} \nu_{2}=n_{2} \nu_{\Lambda} .
$$

$$
\mathrm{N}_{1} \nu_{1}+\mathrm{N}_{2} \nu_{2}=\left(n_{1}+n_{2}\right) \nu_{\Delta} \text {. }
$$

But if $\nu_{1}$ and $\nu_{2}$ are not very different, we may replace either frequency by the arithmetic mean $\frac{1}{2}\left(\nu_{1}+\nu_{2}\right)$, and so obtain

$$
\left(\mathrm{N}_{1}+\mathrm{N}_{2}\right) \frac{\nu_{1}+\nu_{2}}{2} \doteqdot\left(n_{1}+n_{2}\right) \nu_{\mathrm{A}} \text {, }
$$

The observed wave-length for $\mathrm{KI}$ is $96 \cdot 7 \mu$, so that we have $\frac{1}{2}\left(\nu_{1}+\nu_{2}\right)=3.10 \times 10^{12}$ sec. $^{-1}$.

For $\mathrm{N}_{1}+\mathrm{N}_{2}$ we may substitute $\mathrm{N}$ the " molecular number." Taking $N_{1}=19$ and $N_{2}=53$, the product is found to be $11 \times 20.3 \times 10^{12}$, which is in satisfactory agreement with the suggested relation.

* Rubens and Hollnagel, loc. cit.

$\dagger$ Rubens and Hollnagel, loc. cit. 
AgCl. The average wave-length of the residual radiation* was found to be $81.5 \mu\left(\nu=3.68 \times 10^{12}\right)$. The value of $\left(\mathrm{N}_{1}+\mathrm{N}_{2}\right) \nu=11 \times 21.5 \times 10^{12}$.

$\mathbf{P b C l}_{2}$. The average wave-length in this case $*$ was $91 \cdot 0 \mu\left(\nu=3 \cdot 30 \times 10^{12}\right)$, giving $\left(\mathrm{N}_{1}+2 \mathrm{~N}_{2}\right) \nu=18 \times 21 \cdot 2 \times 10^{12}$. When such large integers are involved, it is always possible to find an integer which will give a concordant value for $\nu_{\Delta}$.

$\mathrm{HgCl}$. The average wave-length observed ${ }^{*}$ was $98 \cdot 8 \mu$ $\left(v=3.04 \times 10^{12}\right)$. Taking $\mathrm{N}_{1}=80, \mathrm{~N}_{2}=17$, we find

$$
\left(\mathrm{N}_{1}+\mathrm{N}_{2}\right) \nu=14 \times 21.0 \times 10^{12} \text {. }
$$

AgBr. For this salt ${ }^{*} \lambda=112 \cdot 7 \mu\left(\nu=2 \cdot 66 \times 10^{12}\right)$, and $\left(\mathrm{N}_{1}+\mathrm{N}_{2}\right) \nu=10 ! 2 \times 20.8 \times 10^{12}$.

$\mathrm{CaCO}_{3}$. The case of calc spart is interesting as it contains three elements, whilst only two bands have been recorded, a strong band at $93 \cdot() \mu\left(\nu=3 \cdot 23 \times 10^{12}\right)$ and a weaker at $116 \cdot 1 \mu\left(\nu=2.58 \times 10^{12}\right)$. It is found that the strong band must be assigned to calcium, giving $\mathrm{N} \nu=3 \times 21.5 \times 10^{12}$, the weaker to oxygen, giving $\mathrm{N}_{\nu}=1 \times 20 \cdot 7 \times 10^{12}$.

In a later paper Rubens and Wartenberg $\ddagger$ have given the wave-lengths for two ammonium salts and three compounds of thallium.

$\mathrm{NH}_{4} \mathrm{Cl}$. 'The observed wave-lengths are very nearly the same as those for $\mathrm{NaCl}$, being $54 \cdot 0 \mu\left(\nu=5.56 \times 10^{12}\right)$ and $46.3 \mu \quad\left(\nu=6.48 \times 10^{12}\right)$ respectively. This is what might be expected if the group $\mathrm{NH}_{4}$ be regarded as a compound radicle replacing $\mathrm{Na}$, for the "molecular number" of ammonium $(7+4)$ is the same as the atomic number of sodium (11). Taking the first line as associated with the $\mathrm{NH}_{4}$ group, $\mathrm{N} \nu=3 \times 20 \cdot 4 \times 10^{12}$, whilst the second line gives for the chlorine atom $\mathrm{N}_{\nu}=5 \times 22 \cdot 0 \times 10^{12}$.

$\mathrm{NH}_{1} \mathrm{Br}$. Here again two wave-lengths have been observed, $62 \cdot 3 \mu \quad\left(\nu=4 \cdot 82 \times 10^{12}\right)$ and $55 \cdot 3 \mu \quad\left(\nu=5 \cdot 42 \times 10^{12}\right)$. The former line must be associated with bromine, giving $\mathrm{N} \nu=8 \times 21.1 \times 10^{12}$; the latter with the ammonium group, yielding $\mathrm{N} \nu=3 \times 20^{\circ} 0 \times 10^{12}$.

TICl. The mean wave-lengtll recorded is $91 \cdot 6 \mu(\nu=3.27$ $\left.\times 10^{12}\right)$. The atomic number of thallium being 81 and that of chlorine 17, we find $\mathrm{N} \nu=15 \times 21.4 \times 10^{12}$.

TlBr. For the bromide the mean wave-length is $117 \cdot 0 \mu$ $\left(\nu=2.56 \times 10^{12}\right)$. In this case we find $\mathrm{N} \nu=14 \times 21.2 \times 10^{12}$.

TII. For the iodide the mean wave-length is $151.8 \mu$ $\left(y=1.98 \times 10^{12}\right)$, giving for $\mathrm{N} \nu$ the value $13 \times 20.4 \times 10^{12}$.

* Rubens, Preuss. Akad. Berlin, vol. xxviii. p. 513 (1913).

$\dagger$ Rubens, D. P. G. V. vol. xiii. p. 102 (1911).

$\ddagger$ Rubens and Wartenberg, Preuss. Akad. Berlin, p. 169 (1914). 
Thus for the halogen derivatives of thallium the interesting result is found that the "frequency numbers" $(15,14,13)$ diminish by unity in passing from chloride to bromide and from bromide to iodide.

It must be stated that the suggestion which attributes the two absorption bands of $\mathrm{NaCl}, \mathrm{KCl}, \mathrm{KBr}$ to separate atoms is not a new one*. Nernst is of opinion that it was a mere coincidence-" a very curious and misleading one indeed"that calculations of the specific heat on that supposition gave quite good results. Rubens was unable to find two bands in the case of $\mathrm{AgCl}$ and $\mathrm{PbCl}_{2}$, and came to the conclusion that the two apparent bands were due simply to water vapour, which has a great number of absorption bands. If this conclusion be accepted the results quoted above will require modification in the sense that the "molecular number " must be employed instead of the atomic number; but the proposed relation will still hold good. Thus for $\mathrm{NaCl}$ we find $\mathrm{N} \nu=8 \times 20^{\circ} 6 \times 10^{12}$, for $\mathrm{KCl} N \nu=8 \times 20 \cdot 1 \times 10^{12}$, and for $\mathrm{KBr} \mathrm{N} \nu=9 \times 21 \cdot 6 \times 10^{12}$.

It would appear probable that the relation here discussed applies only in the case of the longer residual rays, having a wave-length greater than siy $20 \mu$. For shorter wavelengths, corresponding to a higher frequency, the value of the product, $\mathrm{N \nu}$, is so large that no real test of the proposed relation can be obtained. It is unfortunate for our present purpose that although the residual rays from quartz in the region of $9 \mu$ and $13 \mu$ have been measured with considerable aceuracy, the longer waves have not as yet been determined accurately; we knuw only that quartz shows strong selective. absorption for the region between 60 and $80 \mu$.

It may be worthy of mention that water vapour has an absorption band at $14: 3 \mu$, which is the wave-length corresponding to a frequency $21.0 \times 10^{12} \mathrm{sec}^{-1}$, that is the frequency here denoted by $\nu_{\Lambda}$. Further, the vapour of carbon dioxide has an absorption hand at $14 \cdot 1 \mu$. It would be of interest to know whether other substances show absorption in the same region.

The possibility of deducing the wave-length of the infra-red radiation from the elastic properties of the solid has been discussed by Madelung and by Sutherland. By considering a cubical space-lattice the former obtained for the wave-length the expression

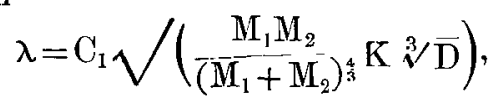

* Cf. Nernst, 'The Theory of the Solid State,' p. 80 (1914). 
where $M_{1}, M_{2}$ are the masses of the atoms, $K$ is the compressibility, and D the density. This may be compared with Einstein's formula. Rubens and Wartenberg have shown that this formula gives results in moderately good agreement with their observations.

They have also obtained fair agreement by employing a modified form of the equation of Lindemann, viz.

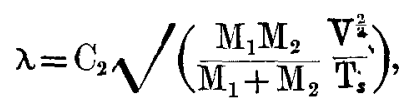

where $\mathrm{V}$ is the molecular volume and $\mathrm{T}_{s}$ the meltingpoint.

In both formulæ the constant must be determined empirically.

The results of this and preceding papers support the following conclusions:-

(1) The forces binding the atoms in the molecule are similar in character to those which bind the molecules of the solid, that is the forces of chemical affinity are of the same nature as the forces of molecular cohesion*.

(2) There must be something of a discrete character in the nature of these forces, in order to account for the occurrence of integral values of $n$. The simplest hypothesis is to assume that the forces arise from the presence of valency electrons. As it is probable that these forces act only in definite directions, it is a plausible suggestion that the linkages between the atoms are constituted by Faraday tubes of force, which would then be regarded as physical entities. The fundamental frequencies, $\nu_{A}$ and $\nu_{E}$, would depend on the properties of the unit tube of force. It has been pointed out by Prof. Nicholson $†$ that such a view seems to be required in order to explain the relations between the frequencies of spectral series. Attention may also be drawn to an important article by Sir J. J. Thomson on the Forces between Atoms and Chemical Affinity $\ddagger$, in which chemical valency is discussed from the same standpoint.

* Compare Nernst, 'The Theory of the Solid State,'pp. 4-9 (1914) ;

Langmuir, Ann. Chem. Soc. Journ. vol. xxxviii. p. 2221 (1916).

+ Nicholson, Phil. Mag. vol. xxvii. p. 541, vol. xxviii. p. 90 (1914).

† J. J. Thomson, Phil. Mag. vol, xxvii. p. 757 (1914). 